Article

\title{
Synthesis and Excellent Duplex Stability of Oligonucleotides Containing 2'-Amino-LNA Functionalized with Galactose Units
}

\author{
Rajesh Kumar ${ }^{1,2}$, Annika Ries ${ }^{1}$ and Jesper Wengel ${ }^{1, *}$ \\ 1 Biomolecular Nanoscale Engineering Center, Department of Physics, Chemistry and Pharmacy, \\ University of Southern Denmark, Campusvej 55, 5230 Odense M, Denmark; \\ rajeshenzyme@gmail.com (R.K.); ries.annika@web.de (A.R.) \\ 2 Department of Chemistry, University of Delhi, Delhi 110007, India \\ * Correspondence: jwe@sdu.dk; Tel.: +45-655-025-10 \\ Academic Editor: Harri Lönnberg \\ Received: 3 May 2017; Accepted: 17 May 2017; Published: 21 May 2017
}

\begin{abstract}
A convenient method for the preparation of oligonucleotides containing internally-attached galactose and triantennary galactose units has been developed based on click chemistry between 2 - $N$-alkyne 2 -amino-LNA nucleosides and azido-functionalized galactosyl building blocks. The synthesized oligonucleotides show excellent binding affinity and selectivity towards complementary DNA/RNA strands with an increase in the melting temperature of up to $+23.5^{\circ} \mathrm{C}$ for triply-modified variants.
\end{abstract}

Keywords: 2'-Amino-LNA; click chemistry; duplex stability; triantennary galactose ligand; oligonucleotides

\section{Introduction}

Nucleic acids have attracted ample interest in the areas of nanotechnology, biology, and biochemistry over the past decade [1-4]. Oligonucleotide (ON) chemistry has been developed with the aim of modifying the sugar ring [5,6], the nucleobases [7-9], or the backbone [10,11], and numerous studies have highlighted the importance of the furanose ring conformation on the biophysical properties of ONs [12-17]. Locked nucleic acid (LNA, Figure 1) [18,19] is a very promising candidate of this class of compounds as the incorporation of a LNA monomer into ONs has been reported to induce excellent duplex stability, favorable mismatch discrimination, and improved resistance to nucleolytic digestion [20-24]. The bicyclic skeleton which "locks" the furanose ring of LNA nucleotides into an RNA mimicking C3'-endo ( $N$-type) conformation is key to these specific properties making LNA-containing oligonucleotides an attractive tool for diagnostic and therapeutic applications [25-29]. As a close analog to LNA, 2'-amino-LNA (Figure 1) [30,31] has been developed to enable incorporation of additional functional groups along the minor groove of high-affinity ONs [32], and recently it was shown that incorporation of 2 '-amino-LNA monomers can improve the therapeutic potential of siRNA and aptamer constructs [33-35].

Several research groups have during the last years been involved in the synthesis of ONs conjugated with galactose (Gal) and $N$-acetylgalactosamine (GalNAc) units as asialoglycoprotein receptor (ASGPR) ligands [36-43]. As one example, GalNAc-conjugated siRNAs targeting TTR (transthyretin) mRNA has been reported to show potent activity in human liver and to reduce circulating TTR protein levels in the blood [44]. Likewise, Østergaard et al. [45,46] demonstrated that 5 '-conjugated triantennary GalNAc antisense oligonucleotides (ASOs) act as a hepatocyte targeting pro-drug which can be metabolized to liberate the parent ASO inside liver cells, and similar targeting has been reported using conjugated siRNAs [47-49]. 
Among multiple DNA linking strategies developed to date, the copper(I)-catalyzed azide alkyne cycloaddition (CuAAC) reaction is a robust and highly adaptable bioconjucation process ("click reactions") for ONs allowing preparation of various conjugates in high yields [50-53], and single to triple modification of oligonucleotides by this reaction has been reported [54-59].

Herein, we report synthesis and characterization of ONs modified via copper(I)-catalyzed click chemistry with galactose derivatives $\mathbf{M}^{2}$ and $\mathbf{M}^{3}$.
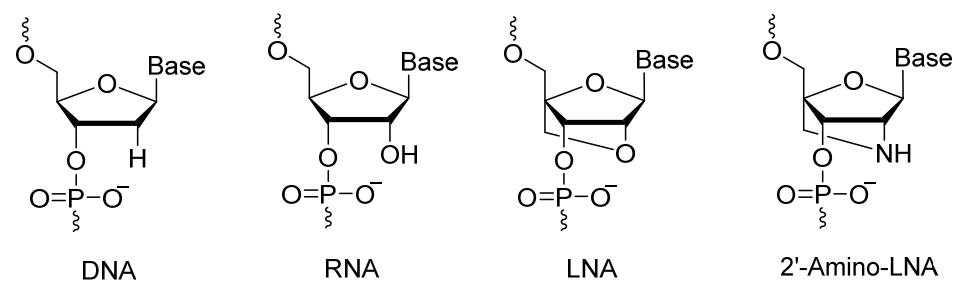

Figure 1. Chemical structure of DNA, RNA, LNA, and 2'-amino-LNA nucleotide monomers.

\section{Results and Discussion}

\subsection{Synthesis of Azido-Functionalized Galactose Derivatives}

2-[2-(2-Azidoethoxy)ethoxy]ethoxy-2,3,4,6-tetra-O-acetyl- $\beta$-D-galactopyranoside (1) and 2-[2-(2-azidoethoxy)ethoxy]ethoxy- $\beta$-D-galactopyranoside (2) were synthesized according to procedures in the literature [60,61]. For synthesis of compound 3 we first tried the reduction of azido group 1 into an amino group with $10 \% \mathrm{Pd} / \mathrm{C}$ in different organic solvents (MeOH/EtOH/EtOAc) in an $\mathrm{H}_{2}$ atmosphere. We observed in every case intramolecular acetyl migration from the sugar to the amine. To avoid this acetyl migration the amine was directly equipped with a Boc-protecting group after azide reduction with $\mathrm{Pd} / \mathrm{C}$ in EtOAc furnishing galactopyranoside 3 in 79\% yield (Scheme 1).

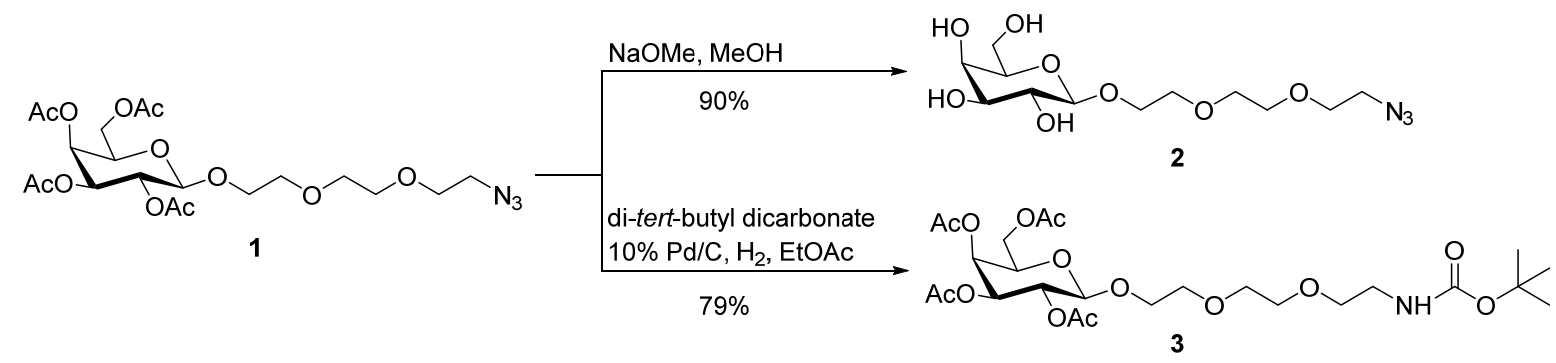

Scheme 1. Synthesis of galactopyranoside intermediates.

The amino group of compound 4 [62] was subsequently reacted with 2-azidoacetic acid in $N, N$-dimethylformamide/tetrahydrofuran (DMF/THF) in the presence of 1-[bis(dimethylamino) methylene]-1H-1,2,3-triazolo-[4,5-b]pyridinium 3-oxide hexafluorophosphate (HATU) and $N, N$-diisopropylethylamine (DIPEA) to afford azido derivative $\mathbf{5}$ in $76 \%$ yield. The ester groups were next converted into the corresponding acids to give 6 , followed by reaction with pentafluorophenol (PFP) in dichloromethane (DCM) in the presence of $N, N$-diisopropylcarbodiimide (DIC) to afford tris-pentafluorophenol ester 7 in 77\% yield. The Boc-protecting group of galactopyranoside 3 was removed by standard conditions (1:3 trifluoroacetic acid (TFA)/DCM, $1 \mathrm{~h}$ ) yielding the corresponding amino galactopyranoside intermediate 3a. Further reaction with tris-pentafluorophenol ester 7 furnished the triantennary compound 8 in $69 \%$ yield, which upon ester cleavage was converted into the desired triantennary azido galactopyranoside 9 in 75\% yield (Scheme 2). 


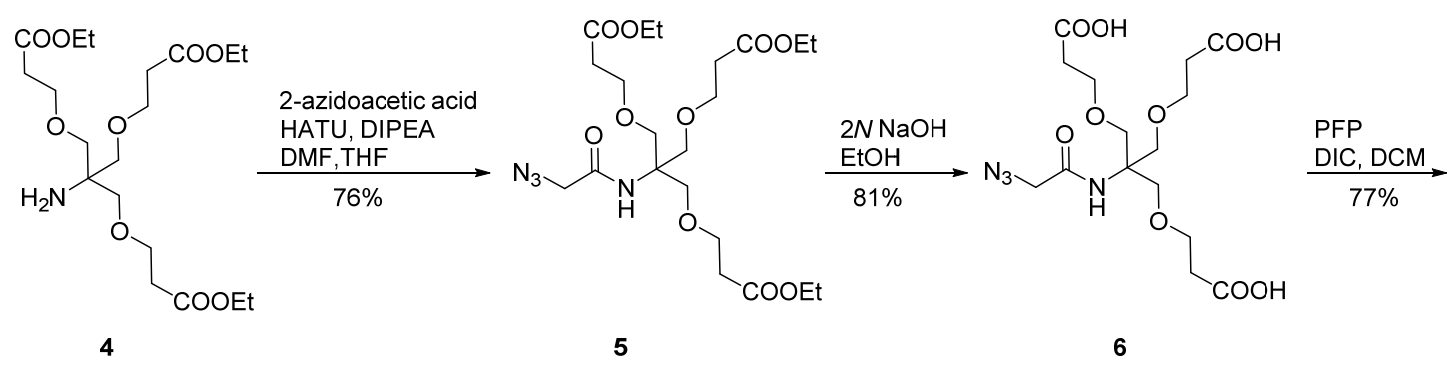<smiles>CCCOC(=O)CCOCC(COCCC(=O)OP)(COCCC(=O)OCCP)NC(=O)CN</smiles>

7

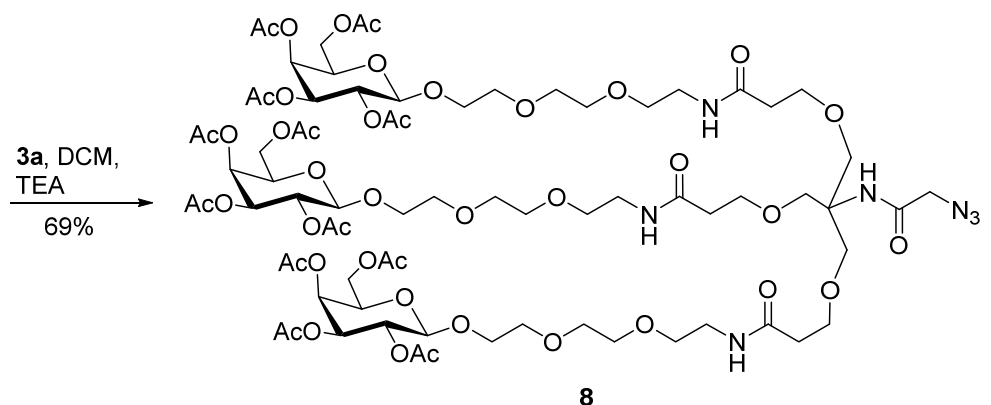

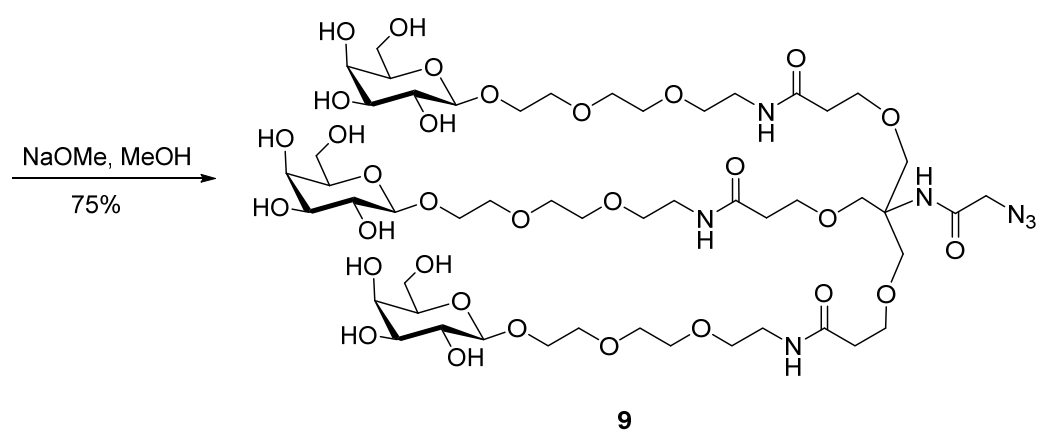

Scheme 2. Synthesis of triantennary azido galactopyranoside.

\subsection{ON Synthesis}

Synthesis of 9-mer ONs was performed at a $1.0 \mu \mathrm{mol}$ scale using standard solid-phase phosphoramidite chemistry on an automated DNA synthesizer. Phosphoramidite building block 10 [54] showed a satisfactory step-wise coupling yield of $>94 \%$ using $1 \mathrm{H}$-tetrazole as the activator and a coupling time of $20 \mathrm{~min}$ used for incorporation of monomer $\mathbf{M}^{\mathbf{1}}$ into ONs, one or three times according

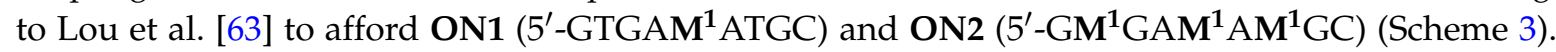
The synthesized ONs were passed through NAP-10 column, and their purity $(>90 \%)$ and composition was confirmed by ion-exchange (IE) HPLC and MALDI-MS (see Section 3.8), respectively. 


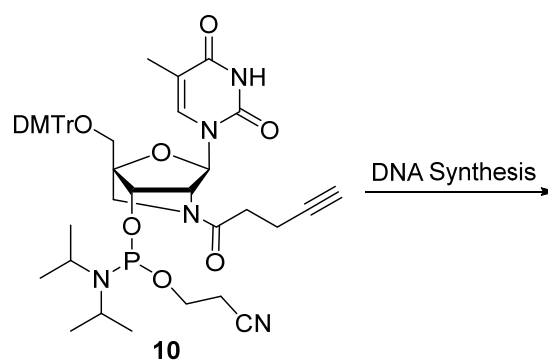

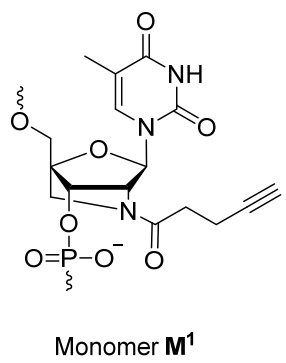

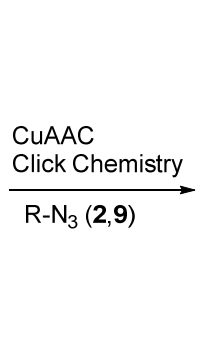

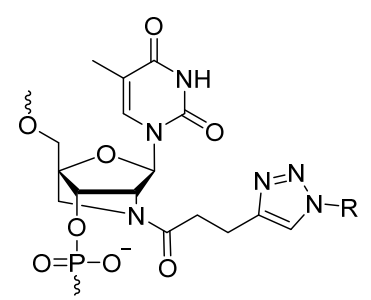

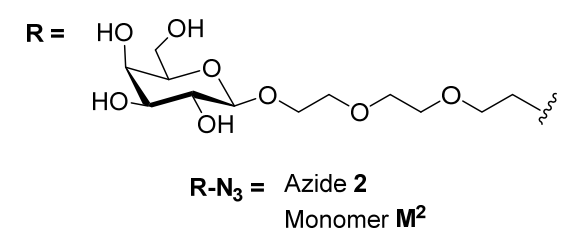

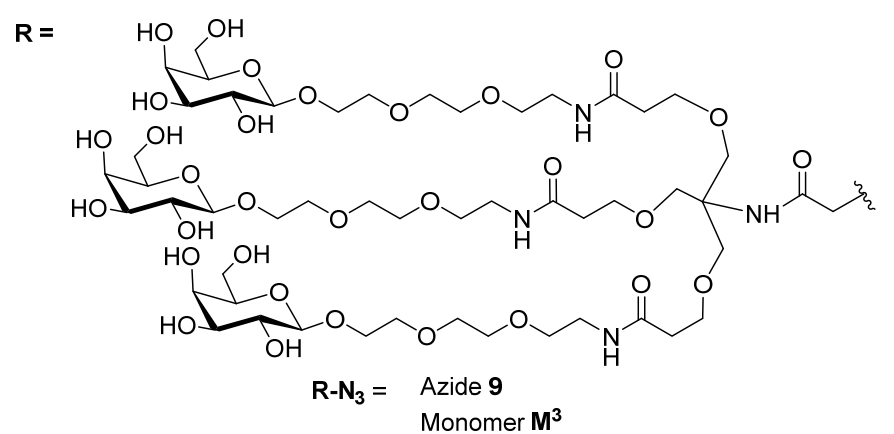

Scheme 3. Synthesis of alkynyl 2'-amino-LNA monomer $\mathbf{M}^{\mathbf{1}}$ and incorporation of monomers $\mathbf{M}^{\mathbf{2}}$ and $\mathbf{M}^{3}$ into ONs.

\subsection{Post-Oligomerization CuAAC Click Chemistry}

Post-oligomerization (i.e., after completion of $\mathrm{ON}$ synthesis and purification) azide-alkyne click chemistry on ON1 and ON2 was accomplished with mono and triantennary azido galactopyranosyl derivatives 2 and 9 under microwave conditions. Dimethyl sulfoxide (DMSO), 2 Mtriethylammonium acetate buffer ( $\mathrm{pH} 7.4$ ), azides 2 or $\mathbf{9}$, copper(II)sulfate-tri(benzyltriazolylmethyl)amine (TBTA) (1:1) and ascorbic acid were added to a deaerated aqueous solution of the starting ON. The purity $(>90 \%)$ and composition of the products ON3-ON6 was confirmed by IE HPLC and MALDI-MS (see Section 3.10), respectively.

\subsection{Thermal Denaturation Studies}

\subsubsection{Binding Affinity}

The effect upon incorporation of one (ON3 and ON5) or three (ON4 and ON6) galactopyranosyl modifications into mixed sequence 9-mer ONs on the thermal stability and base-pairing specificity of duplexes with DNA and RNA complements was evaluated by UV-VIS thermal denaturation experiments (Tables 1 and 2). All changes in thermal denaturation temperatures $\left(T_{\mathrm{m}}\right)$ of modified nucleic acid duplexes are discussed relative to the $T_{\mathrm{m}}$ values measured for the unmodified reference duplexes.

ON7 and ON8 containing one or three non-conjugated 2'-amino-LNA-T monomers showed a substantially increased thermal affinity towards DNA $\left(\Delta T_{\mathrm{m}}\right.$ value $+9.5^{\circ} \mathrm{C}$ with three monomers $\left.\mathbf{M}^{4}\right)$ and RNA complementary strands $\left(\Delta T_{\mathrm{m}}\right.$ value $+20.0{ }^{\circ} \mathrm{C}$ with three monomers $\left.\mathbf{M}^{4}\right)$ (Table 1$)$. Remarkably, the corresponding ONs containing one or three monomer(s) $\mathbf{M}^{2}$ or $\mathbf{M}^{3}$ (ON3-ON6) display similar increases in thermal affinity, with ON5:DNA (single incorporation of $\mathbf{M}^{3}$ ) as the only exception $\left(\Delta T_{\mathrm{m}}\right.$ value of only $+2.0^{\circ} \mathrm{C}$ (Table 1$\left.)\right)$. These results demonstrate that both the mono- and triantennary galactopyranosyl unit are well tolerated in a short ON aimed at DNA or RNA targeting. 
Table 1. Thermal denaturation temperatures of matched duplexes (DNA/DNA and DNA / RNA) ${ }^{a}$.

\begin{tabular}{|c|c|c|c|}
\hline ON & Sequence & $\begin{array}{c}\text { DNA: } 3^{\prime} \text {-CACTATACG } \\
T_{\mathrm{m}} / \Delta T_{\mathrm{m}}\left({ }^{\circ} \mathrm{C}\right)\end{array}$ & $\begin{array}{c}\text { RNA: } 3^{\prime} \text {-CACUAUACG } \\
T_{\mathrm{m}} / \Delta T_{\mathrm{m}}\left({ }^{\circ} \mathrm{C}\right)\end{array}$ \\
\hline $\mathrm{ON}^{\mathrm{ref}}$ & $5^{\prime}$-GTGATATGC & 29.0 & 27.0 \\
\hline ON3 & $5^{\prime}$-GTGAM ${ }^{2}$ ATGC & $33.0 /+4.0$ & $35.5 /+8.5$ \\
\hline ON4 & $5^{\prime}-\mathrm{GM}^{2} \mathrm{GAM}^{2} \mathrm{AM}^{2} \mathrm{GC}$ & $40.0 /+11.0$ & $50.5 /+23.5$ \\
\hline ON5 & $5^{\prime}$-GTGAM ${ }^{3}$ ATGC & $31.0 /+2.0$ & $34.5 /+7.5$ \\
\hline ON6 & $5^{\prime}-\mathrm{GM}^{3} \mathrm{GAM}^{3} \mathrm{AM}^{3} \mathrm{GC}$ & $37.5 /+8.5$ & $48.5 /+21.5$ \\
\hline ON7 & $5^{\prime}$-GTGAM ${ }^{4}$ ATGC & $33.0 /+4.0$ & $35.0 /+8.0$ \\
\hline ON8 & $5^{\prime}-\mathrm{GM}^{4} \mathrm{GAM}^{4} \mathrm{~A} \mathbf{M}^{4} \mathrm{GC}$ & $38.5 /+9.5$ & $47.0 /+20.0$ \\
\hline
\end{tabular}

a Thermal denaturation temperature $T_{\mathrm{m}}\left({ }^{\circ} \mathrm{C}\right)$ (change in $T_{\mathrm{m}}$ relative to corresponding reference duplex $\mathrm{ON}^{\text {ref }}$ :DNA/RNA, $\left.\Delta T_{\mathrm{m}}\left({ }^{\circ} \mathrm{C}\right)\right) . T_{\mathrm{m}}$ values measured as the maximum of the first derivatives of the melting curves ( $\mathrm{A}_{260}$ vs. temperature) using $1.0 \mu \mathrm{M}$ concentration of complementary strand. UV-VIS thermal denaturation experiments performed in a medium salt buffer [ $\mathrm{NaCl}(100 \mathrm{mM})$, EDTA $(0.1 \mathrm{mM}), \mathrm{NaH}_{2} \mathrm{PO}_{4}(10 \mathrm{mM}), \mathrm{Na}_{2} \mathrm{HPO}_{4}$ $(5 \mathrm{mM}), \mathrm{pH} 7.0$ ] with reported $T_{\mathrm{m}}$ values being averages of at least two measurements within $\pm 0.5^{\circ} \mathrm{C}$. $\mathbf{M}^{4}$ is non-conjugated 2'-amino-LNA-T, $\mathbf{M}^{3}$ is triantennary galactopyranosyl-conjugated triazole-linked 2'-amino-LNA, and $\mathbf{M}^{2}$ is monoantennary galactopyranosyl-conjugated triazole-linked 2'-amino-LNA.

Table 2. Thermal denaturation temperatures of mismatched duplexes ${ }^{a}$.

\begin{tabular}{|c|c|c|c|c|c|c|c|c|c|}
\hline \multirow{3}{*}{ ON } & \multirow{3}{*}{ Sequence } & \multicolumn{4}{|c|}{ DNA: 3'-CACTXTACG } & \multicolumn{4}{|c|}{ RNA: $3^{\prime}$-CACUXUACG } \\
\hline & & \multicolumn{2}{|c|}{$T_{\mathrm{m}} /{ }^{\circ} \mathrm{C}$} & \multicolumn{2}{|c|}{$\Delta T_{\mathrm{m}} /{ }^{\circ} \mathrm{C}$} & \multicolumn{2}{|c|}{$T_{\mathrm{m}} /{ }^{\circ} \mathrm{C}$} & \multicolumn{2}{|c|}{$\Delta T_{\mathrm{m}} /{ }^{\circ} \mathrm{C}$} \\
\hline & & A & $\mathbf{T}$ & G & $\mathrm{C}$ & A & $\mathbf{U}$ & G & $\mathrm{C}$ \\
\hline $\mathrm{ON}^{\mathrm{ref}}$ & 5'-GTGATATGC & 29.0 & -15.0 & -9.5 & -16.0 & 27.0 & -16.0 & -5.0 & -21.0 \\
\hline ON3 & $5^{\prime}$-GTGAM ${ }^{2}$ ATGC & 33.0 & -16.0 & -15.0 & -18.0 & 35.5 & -17.5 & -10.5 & -17.5 \\
\hline ON4 & $5^{\prime}-\mathrm{GM}^{2} \mathrm{GAM}^{2} \mathrm{AM}^{2} \mathrm{GC}$ & 40.0 & -15.5 & -17.5 & -18.0 & 50.5 & -16.5 & -11.0 & -15.5 \\
\hline ON5 & $5^{\prime}$-GTGAM ${ }^{3}$ ATGC & 31.0 & -16.5 & -16.0 & -18.0 & 34.5 & -17.5 & -10.0 & -18.0 \\
\hline ON6 & $5^{\prime}-\mathrm{GM}^{3} \mathrm{GAM}^{3} \mathrm{AM}^{3} \mathrm{GC}$ & 37.5 & -15.5 & -15.0 & -18.0 & 48.5 & -15.5 & -10.0 & -15.0 \\
\hline ON7 & $5^{\prime}$-GTGAM ${ }^{4}$ ATGC & 33.0 & -16.0 & -14.5 & -18.5 & 35.0 & -17.0 & -9.0 & -17.0 \\
\hline ON8 & $5^{\prime}-\mathrm{GM}^{4} \mathrm{GAM}^{4} \mathrm{~A} \mathbf{M}^{4} \mathrm{GC}$ & 38.5 & -16.0 & -15.0 & -18.5 & 47.0 & -15.5 & -9.0 & -15.5 \\
\hline
\end{tabular}

\subsubsection{Binding Specificity}

Next, the binding specificities of single- and triple-modified oligonucleotides were evaluated against centrally-positioned mismatched bases (Table 2). We found, in general, very similar pairing selectivity for all sequences $\left(\mathrm{ON}^{\text {ref }}, \mathbf{O N 3}-\mathrm{ON} 8\right)$ with the notable exceptions that the modified strands all showed better discrimination against a DNA or RNA G mismatch than the control, and that the control showed better discrimination against an RNA C mismatch than the modified sequences.

All DNA ON ${ }^{\text {ref }}$ displayed a thermal denaturation temperature of $29.0{ }^{\circ} \mathrm{C}$ with a DNA complementary strand, and an RNA counterpart at $27.0^{\circ} \mathrm{C}$. The results of ON3 showed that the monoantennary galactopyranosyl functionalized triazole-linked 2 '-amino-LNA was well tolerated in a DNA/DNA duplex as the thermal denaturation temperature was comparable with that of $\mathrm{ON}^{\text {ref }}$ (Table 1). A remarkable stabilization at $8.5^{\circ} \mathrm{C}$ was observed with the RNA complement. The corresponding three monoantennary galactopyranosyl units in ON4 was better tolerated as the thermal denaturation temperature was induced by $11.0{ }^{\circ} \mathrm{C}$ against a DNA complement and $23.5^{\circ} \mathrm{C}$ against an RNA complement. In general, similar trends were observed in ON5-ON8 showed increased thermal duplex stability against both DNA and RNA relative to $\mathbf{O N}^{\text {ref, }}$, but the sequence containing a singly-incorporated triantennary galactopyranosyl unit in ON5 stabilized less than the non-conjugated 2'-amino-LNA ON7. Additionally, the corresponding non-conjugated 2'-amino-LNA (ON8) was better tolerated than the triply-incorporated triantennary galactopyranosyl unit in ON6 against a DNA complement, and a reverse trend was observed in an RNA complement, respectively (Table 1). For evaluation of the mismatch discriminative properties of these mono-/triantennary 
galactopyranosyl-conjugated and non-conjugated 2'-amino-LNA ONs were chosen with one mismatched nucleotide opposite the sugar modification. All modifications showed similar, or even enhanced, mismatch discrimination relative to the unmodified strand $\mathbf{O N}^{\text {ref }}$, with the discriminative power generally superior against DNA strands (Table 2).

In summary, singly- or triply-incorporated galactopyranose moieties induced significantly increased duplex stability relative to the reference duplexes, thus expanding the results reported for galactopyranose-modified carbohydrate moieties attached to the 2 '-position of nucleotides via triazole [64] and amide linkages $[65,66]$. The data reported herein, in combination with the remarkable exonuclease resistance induced by other $N$-functionalized 2'-amino-LNA nucleotides [63,65], establish monomers $\mathbf{M}^{2}$ and $\mathbf{M}^{3}$ as promising constituents of future RNA-targeting oligonucleotide drugs.

\section{Materials and Methods}

\subsection{General Information}

All reagents were purchased from Sigma-Aldrich (St. Louis, MO, USA) and used without further purification. Dichloromethane, $\mathrm{N}, \mathrm{N}$-diisopropylethylamine (DIPEA), THF, methanol, $\mathrm{N}, \mathrm{N}$-dimethylformamide (DMF) and pyridine were dried over activated $4 \AA$ molecular sieves, and petroleum ether (PE) and ethylacetate (EtOAc) were used as received. All reactions conducted in anhydrous solvents were carried out under an argon atmosphere. Silica gel column chromatography was performed using Merck Millipore silica gel 60 (0.040-0.063 mm) (Darmstadt, Germany). Thin layer chromatography (TLC) was performed using Merck silica gel 60 F254 (0.22 mm thickness, aluminum-backed) (Darmstadt, Germany). Compounds were visualized at $254 \mathrm{~nm}$ or stained with 5\% sulfuric acid in EtOH. ${ }^{1} \mathrm{H}-\mathrm{NMR}$ spectra were measured at $400 \mathrm{MHz},{ }^{13} \mathrm{C}-\mathrm{NMR}$ spectra were measured at $101 \mathrm{MHz}$, and ${ }^{19} \mathrm{~F}-\mathrm{NMR}$ spectra were measured at $376 \mathrm{MHz}$, all on a Bruker AVANCE III 400 spectrometer (Billerica, MA, USA). Chemical shifts are given in ppm and J values are given in Hz. All assignments for ${ }^{1} \mathrm{H}-\mathrm{NMR}$ and ${ }^{13} \mathrm{C}-\mathrm{NMR}$ have been confirmed by COSY, HSQC, and HMBC. HRMS-ESI spectra were recorded on a Bruker APEX III FT-ICR mass spectrometer (Billerica, MA, USA).

\subsection{Synthesis of 2-[2-(2-Tert-butyloxycarbonylamidoethoxy)ethoxy]ethoxy}

\section{2,3,4,6-tetra-O-acetyl- $\beta$-D-galactopyranoside (3)}

2-[2-(2-Azidoethoxy)ethoxy]ethoxy-2,3,4,6-tetra- $O$-acetyl- $\beta$-D-galactopyranoside $(\mathbf{1}, 500 \mathrm{mg}$, $0.99 \mathrm{mmol})$ was dissolved in EtOAc $(5.0 \mathrm{~mL})$, and 10\% Pd/C $(6.0 \mathrm{mg})$ and di-tert-butyl dicarbonate (324 mg, $1.48 \mathrm{mmol}$ ) were added. The reaction mixture was stirred at r.t. in an $\mathrm{H}_{2}$ atmosphere for $6 \mathrm{~h}$ according to a literature procedure [67]. The reaction mixture was passed through a celite pad and concentrated to dryness under reduced pressure. The residue was purified by silica gel column chromatography using 20-40\% EtOAc in petroleum ether $(v / v)$ as eluent to afford compound 3 as a viscous oil $(451 \mathrm{mg})$ in $79 \%$ yield. $R_{\mathrm{f}}=0.3\left(\right.$ EtOAc/PE, 50:50). ${ }^{1} \mathrm{H}-\mathrm{NMR}\left(400 \mathrm{MHz}, \mathrm{CDCl}_{3}\right): \delta$ $5.39(\mathrm{~d}, J=2.6 \mathrm{~Hz}, 1 \mathrm{H}, \mathrm{H}-4), 5.21(\mathrm{dd}, J=10.5,8.0 \mathrm{~Hz}, 1 \mathrm{H}, \mathrm{H}-2), 5.02(\mathrm{dd}, J=10.4,3.4 \mathrm{~Hz}, 1 \mathrm{H}$, $\mathrm{H}-3), 4.56$ (d, J = 8.0 Hz, 1H, H-1), 4.22-4.07 (m, 2H, H-6a, H-6b), 4.01-3.88 (m, 2H, H-5, $\mathrm{CH}_{2}$ ), 3.78-3.72 (m, $\left.1 \mathrm{H}, \mathrm{CH}_{2}\right), 3.71-3.58\left(\mathrm{~m}, 7 \mathrm{H}, \mathrm{CH}_{2}, \mathrm{NH}\right), 3.54\left(\mathrm{t}, J=5.2 \mathrm{~Hz}, 2 \mathrm{H}, \mathrm{CH}_{2}\right), 3.33-3.30(\mathrm{~m}, 2 \mathrm{H}$, $\mathrm{CH}_{2}$ ), 2.15 (s, 3H, OAc), 2.06 (s, 3H, OAc), 2.05 (s, 3H, OAc), 1.99 (s, 3H, OAc), 1.45 (s, 9H, t-Bu). ${ }^{13} \mathrm{C}-\mathrm{NMR}\left(101 \mathrm{MHz}, \mathrm{CDCl}_{3}\right): \delta$ 170.4, 170.2, 170.1, $169.5\left(4 \times \mathrm{CH}_{3} \mathrm{COO}\right), 156.0(\mathrm{NHCO}), 101.4(\mathrm{C}-1)$, $79.2\left(\mathrm{C}\left(\mathrm{CH}_{3}\right)_{3}\right), 70.9(\mathrm{C}-2), 70.7(\mathrm{C}-3), 70.3,69.1\left(\left(\mathrm{OCH}_{2} \mathrm{CH}_{2}\right)_{2} \mathrm{OCH}_{2}\right), 68.8(\mathrm{C}-5), 67.1(\mathrm{C}-4), 61.3(\mathrm{C}-6)$, $40.4\left(\mathrm{CH}_{2} \mathrm{NH}\right), 28.4\left(\mathrm{C}\left(\mathrm{CH}_{3}\right)_{3}\right), 20.7\left(\mathrm{CH}_{3} \mathrm{COO}\right), 20.7\left(2 \times \underline{\mathrm{CH}}_{3} \mathrm{COO}\right), 20.6\left(\mathrm{CH}_{3} \mathrm{COO}\right)$. HRMS (ESI) calcd. for $\left[\mathrm{C}_{25} \mathrm{H}_{41} \mathrm{NO}_{14} \mathrm{Na}\right]^{+}$: 602.2419; found: 602.2416 .

\subsection{Synthesis of 2-Azidoacetyl-N-\{tris[3-(ethylcarboxylethoxy)methyl]methyl\}-amine (5)}

To a solution of amine 4 [62] (1.0 g, $2.37 \mathrm{mmol})$ and 2-azidoacetic acid (310 $\mathrm{mg}, 3.06 \mathrm{mmol})$ in THF $(30 \mathrm{~mL})$ at r.t. were added DIPEA $(0.54 \mathrm{~mL}, 3.08 \mathrm{mmol})$ and HATU $(1.17 \mathrm{~g}, 3.07 \mathrm{mmol}$ in $10 \mathrm{~mL}$ DMF). The reaction mixture was stirred for $6 \mathrm{~h}$ whereupon EtOAc $(50 \mathrm{~mL})$ was added. Washings 
were performed using saturated aq. $\mathrm{NaHCO}_{3}(3 \times 50 \mathrm{~mL})$ and brine $(3 \times 50 \mathrm{~mL})$, and the organic phases were dried $\left(\mathrm{Na}_{2} \mathrm{SO}_{4}\right)$, filtered, and evaporated to dryness under reduced pressure. The residue was purified by silica gel column chromatography $0-2 \% \mathrm{MeOH}$ in $\mathrm{CH}_{2} \mathrm{Cl}_{2}(v / v)$ as eluent to afford compound 5 as a viscous oil $(0.91 \mathrm{~g})$ in $76 \%$ yield. $R_{\mathrm{f}}=0.6\left(\mathrm{MeOH} / \mathrm{CH}_{2} \mathrm{Cl}_{2}, 5: 95\right) .{ }^{1} \mathrm{H}-\mathrm{NMR}(400 \mathrm{MHz}$, $\left.\mathrm{CDCl}_{3}\right): \delta 6.56(\mathrm{brs}, 1 \mathrm{H}, \mathrm{NH}), 4.15\left(\mathrm{q}, J=7.2 \mathrm{~Hz}, 6 \mathrm{H}, \mathrm{CH}_{2}\right), 3.85\left(\mathrm{~s}, 2 \mathrm{H}, \mathrm{CH}_{2}\right), 3.78-3.62\left(\mathrm{~m}, 12 \mathrm{H}, \mathrm{CH}_{2}\right)$, $2.54\left(\mathrm{t}, J=6.2 \mathrm{~Hz}, 6 \mathrm{H}, \mathrm{CH}_{2}\right), 1.27\left(\mathrm{t}, J=7.1 \mathrm{~Hz}, 9 \mathrm{H}, \mathrm{CH}_{3}\right) .{ }^{13} \mathrm{C}-\mathrm{NMR}\left(101 \mathrm{MHz}, \mathrm{CDCl}_{3}\right): \delta 171.6\left(\mathrm{CO}_{2} \mathrm{Et}\right)$,

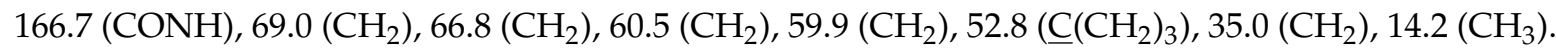
HRMS (ESI) calcd. for $\left[\mathrm{C}_{21} \mathrm{H}_{36} \mathrm{~N}_{4} \mathrm{O}_{10} \mathrm{Na}\right]^{+}$: 527.2324; found: 527.2320.

\subsection{Synthesis of 2-Azidoacetyl-N-\{tris[(2-carboxyethoxy)methyl]methyl\}amine (6)}

Compound 5 (500 mg, $1.19 \mathrm{mmol})$ was dissolved in ethanol $(5.0 \mathrm{~mL})$ and aq. $\mathrm{NaOH}(2 \mathrm{~N}$, $1 \mathrm{~mL}$ ) was added. The reaction mixture was stirred at r.t. for $6 \mathrm{~h}$, concentrated to half value by evaporation under reduced pressure, adjusted to $\mathrm{pH} 3$ with aq. $\mathrm{HCl}(1 \mathrm{~N}, 2 \mathrm{~mL})$, and extracted with EtOAc $(100 \mathrm{~mL})$. The organic phase was dried $\left(\mathrm{Na}_{2} \mathrm{SO}_{4}\right)$, filtered and evaporated to dryness under reduced pressure to afford compound 6 as an off-white solid material $(340 \mathrm{mg})$ in $81 \%$ yield. $R_{\mathrm{f}}=0.5$ (MeOH/ $\left.\mathrm{CH}_{2} \mathrm{Cl}_{2} / \mathrm{CH}_{3} \mathrm{COOH}, 2: 18: 1\right) .{ }^{1} \mathrm{H}-\mathrm{NMR}\left(400 \mathrm{MHz}, \mathrm{DMSO}-d_{6}\right): \delta 12.17$ (brs, 3H, COOH), 7.35 (brs, $1 \mathrm{H}, \mathrm{NH}), 3.76\left(\mathrm{~s}, 2 \mathrm{H}, \mathrm{CH}_{2}\right), 3.67-3.48\left(\mathrm{~m}, 12 \mathrm{H}, \mathrm{CH}_{2}\right), 2.43\left(\mathrm{t}, J=6.3 \mathrm{~Hz}, 6 \mathrm{H}, \mathrm{CH}_{2}\right) .{ }^{13} \mathrm{C}-\mathrm{NMR}$ $\left(101 \mathrm{MHz}, \mathrm{DMSO}-d_{6}\right): \delta 172.5(3 \times \mathrm{COOH}), 167.2(\mathrm{CONH}), 67.9\left(\mathrm{CH}_{2} \mathrm{O}\right), 66.6\left(\mathrm{CH}_{2} \mathrm{O}\right), 59.9\left(\mathrm{C}_{\left(\mathrm{CH}_{2}\right)}\right)$, $50.7\left(\mathrm{CH}_{2} \mathrm{~N}_{3}\right), 34.5\left(\mathrm{CH}_{2} \mathrm{COOH}\right)$. HRMS (ESI) calcd. for $\left[\mathrm{C}_{15} \mathrm{H}_{24} \mathrm{~N}_{4} \mathrm{O}_{10} \mathrm{Na}\right]^{+}$: 443.1385; found: 443.1400 .

\subsection{Synthesis of 2-Azidoacetyl-N-\{tris[3-(pentafluorophenylcarboxylethoxy)methyl]methyl\}amine (7)}

Compound 6 (470 mg, $1.12 \mathrm{mmol})$ was dissolved in $\mathrm{CH}_{2} \mathrm{Cl}_{2}(7.0 \mathrm{~mL})$ and pentafluorophenol $(1.02 \mathrm{~g}, 5.59 \mathrm{mmol})$ was added. The reaction mixture was cooled to $0{ }^{\circ} \mathrm{C}$ and $N, N^{\prime}$-diisopropylcarbodimide $(0.98 \mathrm{~mL}, 6.26 \mathrm{mmol})$ was added, and the reaction mixture was stirred for $6 \mathrm{~h}$ at r.t. The solvent was removed under reduced pressure and the residue was purified by silica gel column chromatography using EtOAc/PE (5-20\%, v/v) as eluent to afford compound 7 as a viscous oil $(789 \mathrm{mg})$ in $77 \%$ yield. $R_{\mathrm{f}}=0.5(\mathrm{EtOAc} / \mathrm{PE}, 2: 3) .{ }^{1} \mathrm{H}-\mathrm{NMR}\left(400 \mathrm{MHz}, \mathrm{CDCl}_{3}\right): \delta 6.47$ (brs, $\left.1 \mathrm{H}, \mathrm{NH}\right), 4.10-3.49(\mathrm{~m}, 14 \mathrm{H}$, $\left.\mathrm{CH}_{2} \mathrm{O}, \mathrm{CH}_{2} \mathrm{~N}_{3}\right), 2.91\left(\mathrm{t}, J=5.9 \mathrm{~Hz}, 6 \mathrm{H}, \mathrm{CH}_{2} \mathrm{COO}\right) .{ }^{13} \mathrm{C}-\mathrm{NMR}\left(101 \mathrm{MHz}, \mathrm{CDCl}_{3}\right): \delta 167.5$ (COOPFP), $166.9\left(\mathrm{COCH}_{2} \mathrm{~N}_{3}\right), 142.3$ (Ar-C), 139.8 (Ar-C), 139.8-138.7 (Ar-C), 136.6 (Ar-C), $68.9\left(\mathrm{CH}_{2} \mathrm{O}\right), 66.1$ $\left(\mathrm{CH}_{2} \mathrm{O}\right), 59.8\left(\underline{\mathrm{C}}\left(\mathrm{CH}_{2}\right)_{3}\right), 52.8\left(\mathrm{CH}_{2} \mathrm{~N}_{3}\right), 34.2\left(\mathrm{CH}_{2} \mathrm{COOPFP}\right) .{ }^{19} \mathrm{~F}-\mathrm{NMR}\left(376 \mathrm{MHz}, \mathrm{CDCl}_{3}\right): \delta-152.9$ $(\mathrm{dd}, J=21.7,4.2 \mathrm{~Hz}, 6 \mathrm{~F}),-157.8(\mathrm{t}, J=21.7 \mathrm{~Hz}, 3 \mathrm{~F}),-162.09--162.64(\mathrm{~m}, 6 \mathrm{~F})$. HRMS (ESI) calcd. for $\left[\mathrm{C}_{33} \mathrm{H}_{21} \mathrm{~F}_{15} \mathrm{~N}_{4} \mathrm{O}_{10} \mathrm{Na}\right]^{+}$: 941.0910; found: 941.0910.

\subsection{Synthesis of Triantennary Azido 2,3,4,6-Tetra-O-Acetyl- $\beta$-D-Galactopyranoside (8)}

Compound 3 (530 mg, $0.91 \mathrm{mmol}$ ) was dissolved in $\mathrm{CH}_{2} \mathrm{Cl}_{2} /$ trifluoroacetic acid (3:1, $12 \mathrm{~mL}$ ) and stirred at r.t. for $1 \mathrm{~h}$. The solvents were evaporated under reduced pressure and the resulting oil was dissolved in anhydrous $\mathrm{CH}_{2} \mathrm{Cl}_{2}(24 \mathrm{~mL})$. To this mixture was added $\mathrm{N}$-\{tris[3-(pentafluorophenylcarboxylethoxy)methyl]methylamide\}-2-azidoacetamide $(7,210 \mathrm{mg}$, $0.23 \mathrm{mmol}$ ) and the resulting mixture was adjusted to $\mathrm{pH} 8$ with triethylamine (TEA) and stirred at r.t. for $10 \mathrm{~h}$. The solvents were removed under reduced pressure and the residue was redissolved in $\mathrm{CH}_{2} \mathrm{Cl}_{2}(50 \mathrm{~mL})$ and washed with $\mathrm{H}_{2} \mathrm{O}(3 \times 50 \mathrm{~mL})$. The separated organic phase was dried $\left(\mathrm{Na}_{2} \mathrm{SO}_{4}\right)$, filtered and evaporated to dryness under reduced pressure. The residue was purified by silica gel chromatography using $\left(\mathrm{MeOH} / \mathrm{CH}_{2} \mathrm{Cl}_{2}, 5: 95 \mathrm{v} / \mathrm{v}\right)$ as eluent to afford the acetyl protected triantennary galactose azide 8 as a white solid material $(285 \mathrm{mg})$ in $69 \%$ yield. $R_{\mathrm{f}}=0.4\left(\mathrm{MeOH} / \mathrm{CH}_{2} \mathrm{Cl}_{2}, 5: 95\right)$. ${ }^{1} \mathrm{H}-\mathrm{NMR}\left(400 \mathrm{MHz}, \mathrm{CDCl}_{3}\right): \delta 6.90(\mathrm{brs}, 1 \mathrm{H}, \mathrm{NH}), 6.59(\mathrm{t}, J=5.5 \mathrm{~Hz}, 3 \mathrm{H}, \mathrm{NH}), 5.39(\mathrm{~d}, J=3.1 \mathrm{~Hz}$, 3H, Gal-H-4), 5.20 (dd, J = 10.4, 8.0 Hz, 3H, Gal-H-2), 5.03 (dd, J = 10.5, 3.4 Hz, 3H, Gal-H-3), 4.56 (d, $J=7.9 \mathrm{~Hz}, 3 \mathrm{H}, \mathrm{Gal}-\mathrm{H}-1), 4.20-4.10(\mathrm{~m}, 6 \mathrm{H}, \mathrm{H}-6), 4.01-3.90\left(\mathrm{~m}, 6 \mathrm{H}, \mathrm{Gal}-\mathrm{H}-5, \mathrm{CH}_{2}\right), 3.88\left(\mathrm{~s}, 2 \mathrm{H}, \mathrm{CH}_{2} \mathrm{~N}_{3}\right)$, 3.78-3.68 (m, $\left.15 \mathrm{H}, \mathrm{CH}_{2} \mathrm{O}\right), 3.68-3.58\left(\mathrm{~m}, 18 \mathrm{H}, \mathrm{CH}_{2} \mathrm{O}\right), 3.56\left(\mathrm{t}, J=5.3 \mathrm{~Hz}, 6 \mathrm{H}, \mathrm{CH}_{2} \mathrm{O}\right), 3.48-3.42(\mathrm{~m}, 6 \mathrm{H}$, $\left.\mathrm{C}_{2} \mathrm{NH}\right), 2.44\left(\mathrm{t}, J=5.8 \mathrm{~Hz}, 6 \mathrm{H}, \mathrm{CH}_{2} \mathrm{CO}\right.$ ), 2.15 (s, 9H, OAc), 2.06 (s, 9H, OAc), 2.05 (s, 9H, OAc), 1.99 (s, $9 \mathrm{H}, \mathrm{OAc}) .{ }^{13} \mathrm{C}-\mathrm{NMR}\left(101 \mathrm{MHz}, \mathrm{CDCl}_{3}\right): \delta 171.2,170.4,170.2,170.1\left(4 \times \mathrm{CH}_{3} \underline{\mathrm{COO}}\right), 169.5\left(\mathrm{CH}_{2} \mathrm{CONH}\right)$, 
$167.2\left(\mathrm{COCH}_{2} \mathrm{~N}_{3}\right), 101.4$ (Gal-C-1), 70.9 (Gal-C-2), 70.7 (Gal-C-3), 70.6, 70.3, 70.1, 69.9, 69.3, 69.1, 67.1 $\left(7 \times \mathrm{CH}_{2} \mathrm{O}\right), 68.9$ (Gal-C-5), 67.5 (Gal-C-4), 61.2 (Gal-C-6), $60.1\left(\underline{\mathrm{C}}\left(\mathrm{CH}_{2}\right)_{3}\right), 52.5\left(\mathrm{CH}_{2} \mathrm{~N}_{3}\right), 39.3\left(\mathrm{CH}_{2} \mathrm{NH}\right)$,

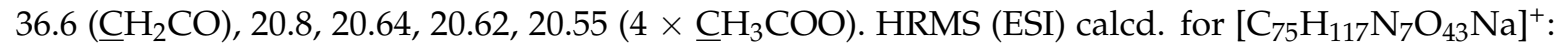
1826.7076; found: 1826.7052 .

\subsection{Synthesis of Triantennary Azido 2- $\beta-\mathrm{D}-$ Galactopyranoside (9)}

Acetyl protected triantennary galactose azide $8(140 \mathrm{mg}, 0.08 \mathrm{mmol})$ was dissolved in anhydrous $\mathrm{MeOH}(20 \mathrm{~mL})$ and NaOMe $(25 \mathrm{wt}$. \% in methanol, $4 \mu \mathrm{L}, 8.0 \mu \mathrm{mol})$ was added. The reaction mixture was stirred at r.t. for $1 \mathrm{~h}$, neutralized with DOWEX-50WX2 ( $\mathrm{H}^{+}$resin), filtered, and evaporated to dryness under reduced pressure. The residue was redissolved in $\mathrm{H}_{2} \mathrm{O}$, dialyzed for two days using Spectra/Por Float-A-Lyzer G2 (MWCO: 100-500 D molecular weight cut-off) and lyophilized to furnish the desired product 9 as a white solid material $(76 \mathrm{mg})$ in $75 \%$ yield; $R_{\mathrm{f}}=0.4\left(\mathrm{CH}_{2} \mathrm{Cl}_{2} / \mathrm{MeOH} / \mathrm{H}_{2} \mathrm{O}\right.$, 1.6:1.0:0.2). ${ }^{1} \mathrm{H}-\mathrm{NMR}\left(400 \mathrm{MHz}, \mathrm{MeOD}-d_{4}\right): \delta 4.30(\mathrm{~d}, J=7.3 \mathrm{~Hz}, 3 \mathrm{H}, \mathrm{Gal}-\mathrm{H}-1), 4.12-3.99$ (m, 3H), 3.9-3.87 (m, 6H), 3.78-3.67 (m, 40H), 3.60-3.50 (m, 17H), $3.42\left(\mathrm{t}, J=5.1 \mathrm{~Hz}, 6 \mathrm{H}, \mathrm{CH}_{2} \mathrm{NH}\right), 2.49(\mathrm{t}$, $\left.J=5.7 \mathrm{~Hz}, 6 \mathrm{H}, \mathrm{CH}_{2} \mathrm{CO}\right) .{ }^{13} \mathrm{C}-\mathrm{NMR}\left(101 \mathrm{MHz}, \mathrm{MeOD}-d_{4}\right): \delta 174.2\left(\mathrm{CH}_{2} \mathrm{CONH}\right), 170.0\left(\mathrm{COCH}_{2} \mathrm{~N}_{3}\right)$, 105.0 (Gal-C-1), 76.7, 74.9, 72.5 (Gal-C-2, Gal-C-3, Gal-C-5), 71.5, 71.2, 70.7, 70.4, 70.1, $69.6\left(6 \times \mathrm{CH}_{2} \mathrm{O}\right)$,

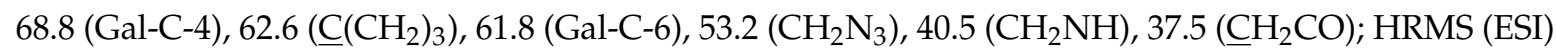
calcd. for $\left[\mathrm{C}_{51} \mathrm{H}_{93} \mathrm{~N}_{7} \mathrm{O}_{31} \mathrm{Na}\right]^{+}: 1322.5808$; found: 1322.5846 .

\subsection{Synthesis and Purification of ON1-ON2}

ONs were synthesized on a DNA synthesizer (PerSpective Biosystems Expedite 8909, (Framingham, MA, USA)) in $1.0 \mu \mathrm{mol}$ scale using manufacturer's standard protocols. For incorporation of monomer $\mathbf{M}^{\mathbf{1}}$ [56] a hand-coupling procedure [68] was used (20 min coupling time and 5-[3,5-bis(trifluoromethyl)phenyl]-1H-tetrazole $\left(0.25 \mathrm{M}\right.$, in anhydrous $\left.\mathrm{CH}_{3} \mathrm{CN}\right)$ as activator). The coupling efficiencies of standard DNA phosphoramidites and phosphoramidite $\mathbf{1 0}$ based on the absorbance of the dimethoxytrityl cation released after each coupling varied between 95\% and $98 \%$. Cleavage from solid support and removal of nucleobase protecting groups was performed using $32 \%$ aq. ammonia for $16 \mathrm{~h}$ at $55^{\circ} \mathrm{C}$. The resulting oligonucleotides were purified by DMT-ON RP-HPLC using Waters System 600 (Milford, MA, USA) equipped with Xterra MS C18-column (5 $\mathrm{mm}, 150 \mathrm{~mm} \times 7.8 \mathrm{~mm}$, (Milford, MA, USA)). Elution was performed starting with an isocratic hold of A-buffer for $5 \mathrm{~min}$ followed by a linear gradient to $70 \%$ B-buffer over $40 \mathrm{~min}$ at a flow rate of $1.0 \mathrm{~mL} / \mathrm{min}$ (A-buffer: $0.05 \mathrm{M}$ triethyl ammonium acetate, $\mathrm{pH} 7.4$; B-buffer: $25 \%$ buffer $\mathrm{A}, 75 \%$ $\left.\mathrm{CH}_{3} \mathrm{CN}\right)$. RP-purification was followed by detritylation (80\% aq. AcOH, $30 \mathrm{~min}$ ) and precipitation (acetone, $-18{ }^{\circ} \mathrm{C}, 12 \mathrm{~h}$ ). The identity and purity of $\mathrm{ON} 1$ and $\mathrm{ON} 2$ were verified by MALDI-TOF mass spectrometry (Billerica, MA, USA) (Table 3) and IE HPLC, respectively. IE HPLC was performed using a Merck Hitachi LaChrom instrument (Tokyo, Japan) equipped with a Dionex DNAPac Pa-100 column (250 $\mathrm{mm} \times 4 \mathrm{~mm}$, Sunnyvale, CA, USA). Elution was performed starting with an isocratic hold of A- and C-buffers for 2 min followed by a linear gradient to 60\% B-buffer over $28 \mathrm{~min}$ at a flow rate of $1.0 \mathrm{~mL} / \mathrm{min}$ (A-buffer: Milli-Q water; B-buffer: $1 \mathrm{M} \mathrm{NaClO}$, C-buffer: $25 \mathrm{mM}$ Tris-Cl, pH 8.0). MALDI-TOF mass-spectrometry was performed using a MALDI-LIFT system on the Ultraflex II TOF/TOF instrument from Bruker (Billerica, MA, USA) using an HPA-matrix (10 mg 3-hydroxypicolinic acid, $50 \mathrm{mM}$ ammonium citrate in $70 \%$ aq. $\mathrm{CH}_{3} \mathrm{CN}$ ).

Table 3. Sequence and MALDI-MS of modified oligonucleotides ON1 and ON2.

\begin{tabular}{cccc}
\hline ON & Sequence & Calculated & Found \\
\hline ON1 & $5^{\prime}-G_{T G A M^{\mathbf{1}} \text { ATGC }}$ & 2861.18 & 2858.69 \\
ON2 & $5^{\prime} \mathrm{GM}^{\mathbf{1}} \mathrm{GAM}^{\mathbf{1}} \mathrm{AM}^{\mathbf{1}} \mathrm{GC}$ & 3075.74 & 3075.78 \\
\hline \multicolumn{4}{c}{$\mathbf{M}^{\mathbf{1}}=2^{\prime}$-alkyne-2'-amino-LNA. }
\end{tabular}




\subsection{Thermal Denaturation Studies}

Thermal denaturation studies were carried out on a Perkin Elmer Lambda 35 UV-VIS spectrometer (Shelton, CT, USA) using a Hellma SUPRASIL synthetic quartz $10 \mathrm{~mm}$ path length cuvettes. The oligonucleotides $(1.0 \mu \mathrm{M}$ per strand) were dissolved in medium salt buffer $[\mathrm{NaCl}(100 \mathrm{mM})$, EDTA $\left.(0.1 \mathrm{mM}), \mathrm{NaH}_{2} \mathrm{PO}_{4}(10 \mathrm{mM}), \mathrm{Na}_{2} \mathrm{HPO}_{4}(5 \mathrm{mM}), \mathrm{pH} 7.0\right]$ and the resulting solution heated to $90{ }^{\circ} \mathrm{C}$ for $10 \mathrm{~min}$ and then slowly cooled down to $5^{\circ} \mathrm{C}$. Concentrations of oligonucleotides were calculated using the extinction coefficients. UV absorbance at $260 \mathrm{~nm}$ as a function of time was recorded and the thermal denaturation temperatures $\left(T_{\mathrm{m}}\right)$ were determined as the maxima of the first derivative of the $U_{2} 60$ vs. temperature curves. The $T_{m}$ values are given as averages of two measurements within $\pm 0.5^{\circ} \mathrm{C}$.

\subsection{Postsynthetic Click Procedure in Solution}

ON ON1 or ON2 (40 nmol) was dissolved in fresh MQ water $(190 \mu \mathrm{L}$ for one incorporation and $151 \mu \mathrm{L}$ for three incorporations) in a $1.0 \mathrm{~mL}$ microwave vial, and $2 \mathrm{M}$ tetra triethylammonium acetate buffer ( $\mathrm{pH} 7.4 ; 20 \mu \mathrm{L}$ ), DMSO $(84 \mu \mathrm{L}$ for one incorporation and $52 \mu \mathrm{L}$ for three incorporations), the corresponding azide 2 or $9(16 \mu \mathrm{L}$ (ON1) for one incorporation and $48 \mu \mathrm{L}$ (ON2) for three incorporations of $10 \mathrm{mM}$ solution in DMSO), $\mathrm{CuSO}_{4}$-TBTA equimolar complex $(10 \mu \mathrm{L}$ of $10 \mathrm{mM}$ stock solution) and ascorbic acid ( $10 \mu \mathrm{L}$ of $25 \mathrm{mM}$ freshly prepared stock solution) were subsequently added under an argon atmosphere. The resulting mixture was deaerated, tightly closed, and subjected to microwave conditions (microwave reactor, $60{ }^{\circ} \mathrm{C}, 2 \mathrm{~h}$, Biotage initiator microwave synthesizer). Thereafter, the reaction was cooled to r.t. and then filtrated through an IIIustra NAP-10 column following manufacture's protocol. The resulting solution was evaporated to dryness at r.t. under a nitrogen atmosphere. The resulting conjugates ON3-ON6 were analyzed by MALDI TOF mass spectrometry (Table 4) and IE HPLC. The ONs (ON4 and ON6) were further purified by RP HPLC. The yields of the click reactions were determined based on the absorbance of the ONs at $260 \mathrm{~nm}: 92 \%$ (ON3), 20\% (ON4), 77\% (ON5), and 17\% (ON6).

Table 4. Sequence and MALDI-MS of conjugated ON3-ON6.

\begin{tabular}{cccc}
\hline ON & Sequence & Calculated & Found \\
\hline ON3 & $5^{\prime}-\mathrm{GTGAM}^{2} \mathrm{ATGC}$ & 3198.33 & 3198.25 \\
ON4 & $5^{\prime}-\mathrm{GM}^{2} \mathrm{GAM}^{2} \mathrm{AM} \mathbf{M}^{2} \mathrm{GC}$ & 4087.19 & 4087.93 \\
ON5 & $5^{\prime}-\mathrm{GTGAM}^{3} \mathrm{ATGC}$ & 4160.77 & 4160.66 \\
ON6 & $5^{\prime}-\mathrm{GM}^{3} \mathrm{GAM}^{3} \mathrm{AM} \mathbf{M}^{3} \mathrm{GC}$ & 6974.51 & 6974.76 \\
\hline
\end{tabular}

$\mathbf{M}^{3}$ is triantennary galactopyranosyl-conjugated triazole-linked $2^{\prime}$-amino-LNA, and $\mathbf{M}^{2}$ is monoantennary galactopyranosyl-conjugated triazole-linked 2'-amino-LNA.

\section{Conclusions}

In summary, we have successfully developed the copper(I)-catalyzed azide alkyne cycloaddition click chemistry conjugation of mono- and triantennary galactospyranosyl units to internally-positioned amino-LNA nucleotides. This method is efficient and provides the desired products in a remarkable purity. High-affinity recognition of complementary DNA and RNA strands has been demonstrated, thus confirming the suitability of the $\mathrm{N} 2{ }^{\prime}$-position $2^{\prime}$-amino-LNA nucleotides for attachment of targeting ligands for therapeutic oligonucleotide constructs.

Acknowledgments: The authors thank The VILLUM Foundation for financial support of The Biomolecular Nanoscale Engineering Centre (BioNEC), grant number VKR022710. A.R. thanks the German Research Foundation (DFG) for a postdoctoral fellowship. Joan Hansen and Tina Grubbe Hansen are thanked for technical support.

Author Contributions: The study was designed by R.K., A.R., and J.W.; and was conducted by R.K., and A.R.; and R.K., A.R., and J.W. contributed to the reagents and materials and data analysis. The paper was written by R.K., A.R., and J.W. All authors approved the final manuscript. 
Conflicts of Interest: The authors declare no conflict of interest.

\section{References}

1. Shabanpoor, F.; McClorey, G.; Saleh, A.F.; Jarver, P.; Wood, M.J.A.; Gait, M.J. Bi-specific splice-switching PMO oligonucleotides conjugated via a single peptide active in a mouse model of Duchenne muscular dystrophy. Nucleic Acids Res. 2015, 43, 29-39. [CrossRef] [PubMed]

2. Sancar, A.; Lindsey-Boltz, L.A.; Unsal-Kacmaz, K.; Linn, S. Molecular mechanisms of mammalian DNA repair and the DNA damage checkpoints. Annu. Rev. Biochem. 2004, 73, 39-85. [CrossRef] [PubMed]

3. Sessler, J.L.; Lawrence, C.M.; Jayawickramarajah, J. Molecular recognition via base-pairing. Chem. Soc. Rev. 2007, 36, 314-325. [CrossRef] [PubMed]

4. $\quad$ Boersma, A.J.; Megens, R.P.; Feringa, B.L.; Roelfes, G. DNA-based asymmetric catalysis. Chem. Soc. Rev. 2010, 39, 2083-2092. [CrossRef] [PubMed]

5. Wilson, C.; Keefe, A.D. Building oligonucleotide therapeutics using non-natural chemistries. Curr. Opin. Chem. Biol. 2006, 10, 607-614. [CrossRef] [PubMed]

6. Prakash, T.P. An overview of sugar-modified oligonucleotides for antisense therapeutics. Chem. Biodivers. 2011, 8, 1616-1641. [CrossRef] [PubMed]

7. Kool, E.T. Replacing the nucleobases in DNA with designer molecules. Acc. Chem. Res. 2002, 35, 936-943. [CrossRef] [PubMed]

8. Wilson, J.N.; Kool, E.T. Fluorescent DNA base replacements: Reporters and sensors for biological systems. Org. Biomol. Chem. 2006, 4, 4265-4274. [CrossRef] [PubMed]

9. Sipa, K.; Sochacka, E.; Kazmierczak-Baranska, J.; Maszewska, M.; Janicka, M.; Nowak, G.; Nawrot, B. Effect of base modifications on structure, thermodynamic stability, and gene silencing activity of short interfering RNA. RNA 2007, 13, 1301-1316. [CrossRef] [PubMed]

10. Chan, J.H.; Lim, S.; Wong, W.S. Antisense oligonucleotides: From design to therapeutic application. Clin. Exp. Pharmacol. Physiol. 2006, 33, 533-540. [CrossRef] [PubMed]

11. Micklefield, J. Backbone modification of nucleic acids: Synthesis, structure and therapeutic applications. Curr. Med. Chem. 2001, 8, 1157-1179. [CrossRef] [PubMed]

12. Meints, G.A.; Karlsson, T.; Drobny, G.P. Modeling furanose ring dynamics in DNA. J. Am. Chem. Soc. 2001, 123, 10030-100038. [CrossRef] [PubMed]

13. Blackburn, G.M.; Gait, M.J.; Loakes, D.; Williams, D.M. DNA and RNA structure. In Nucleic Acids in Chemistry and Biology, 3rd ed.; RCS Publishing: Cambridge, UK, 2006; pp. 13-75.

14. Plashkevych, O.; Chatterjee, S.; Honcharenko, D.; Pathmasiri, W.; Chattopadhyaya, J. Chemical and structural implications of $1^{\prime}, 2^{\prime}$-versus $2^{\prime}, 4^{\prime}$-conformational constraints in the sugar moiety of modified thymine nucleosides. J. Org. Chem. 2007, 72, 4716-4726. [CrossRef] [PubMed]

15. Meldgaard, M.; Wengel, J. Bicyclic nucleosides and conformational restriction of oligonucleotides. J. Chem. Soc. Perkin Trans. 1 2000, 353, 3539-3554. [CrossRef]

16. Leumann, C.J. DNA analogues: From supramolecular principles to biological properties. Bioorg. Med. Chem. 2002, 10, 841-854. [CrossRef]

17. Enderlin, G.; Nielsen, P. Synthesis of $6^{\prime}$-branched locked nucleic acid by a radical TEMPO-scavanged stereoselective mercury cyclization. J. Org. Chem. 2008, 73, 6891-6894. [CrossRef] [PubMed]

18. Singh, S.K.; Koshkin, A.A.; Wengel, J.; Nielsen, P. LNA (locked nucleic acids): Synthesis and high-affinity nucleic acid recognition. Chem. Commun. 1998, 455-456. [CrossRef]

19. Obika, S.; Nanbu, D.; Hari, Y.; Andoh, J.; Morio, K.; Doi, T.; Imanishi, T. Stability and structural features of the duplex containing nucleoside analogues with a fixed $\mathrm{N}$-type conformation, $2^{\prime}-\mathrm{O}, 4^{\prime}$ - $\mathrm{C}$-methyleneribonucleotieds. Tetrahedron Lett. 1998, 39, 5401-5404. [CrossRef]

20. Wengel, J. Synthesis of $3^{\prime}-C$ - and $4^{\prime}$-C-branched oligodeoxynucleotides and the development of locked nucleic acid (LNA). Acc. Chem. Res. 1999, 32, 301-310. [CrossRef]

21. Bell, N.M.; Micklefield, J. Chemical modification of oligonucleotides for therapeutic, bioanalytical and other applications. ChemBioChem 2009, 10, 2691-2703. [CrossRef] [PubMed]

22. Yamamoto, T.; Nakatani, M.; Narukawa, K.; Obika, S. Antisense drug discovery and development. Future Med. Chem. 2011, 3, 339-365. [CrossRef] [PubMed] 
23. Fox, K.R.; Brown, T. Formation of stable DNA triplexes. Biochem. Soc. Trans. 2011, 39, 629-634. [CrossRef] [PubMed]

24. Deleavey, G.F.; Damha, M.J. Designing chemically modified oligonucleotides for targeted gene silencing. Chem. Biol. 2012, 19, 937-954. [CrossRef] [PubMed]

25. Nielsen, K.E.; Singh, S.K.; Wengel, J.; Jacobsen, J.P. Solution structure of an LNA hybridized to DNA: NMR study of the d(CT(L)GCT(L)T(L)CT(L)GC):d(GCAGAAGCAG) duplex containing four locked nucleotides. Bioconjugate Chem. 2000, 11, 228-238. [CrossRef]

26. Jespen, J.S.; Wengel, J. LNA-antisense rivals siRNA for gene silencing. Curr. Opin. Drug Discov. Dev. 2004, 7, 188-194.

27. Frieden, M.; Ørum, H. The application of locked nucleic acids in the treatment of cancer. IDrugs 2006, 9, 706-711. [PubMed]

28. Grunweller, A.; Hartmann, R.K. Locked nucleic acid oligonucleotides: The next generation of antisense agents? Biodrugs 2007, 21, 235-243. [CrossRef] [PubMed]

29. Stenvang, J.; Silahtaroglu, A.N.; Lindow, M.; Fimen, J.; Kauppinen, S. The utility of LNA in microRNA-based cancer diagnostics and therapeutics. Semin. Cancer Biol. 2008, 18, 89-102. [CrossRef] [PubMed]

30. Singh, S.K.; Kumar, R.; Wengel, J. Synthesis of 2'-amino-LNA: A novel conformationally restricted high-affinity oligonucleotide analogue with a handle. J. Org. Chem. 1998, 63, 10035-10039. [CrossRef]

31. Madsen, A.S.; Jørgensen, A.S.; Jensen, T.B.; Wengel, J. Large scale synthesis of 2'-amino-LNA thymine and 5-methylcytosine nucleosides. J. Org. Chem. 2012, 77, 10718-10728. [CrossRef] [PubMed]

32. Astakhova, I.K.; Wengel, J. Scaffolding along nucleic acid duplexes using $2^{\prime}$-amino-locked nucleic acids. Acc. Chem. Res. 2014, 47, 1768-1777. [CrossRef] [PubMed]

33. Gao, Y.; Liu, X.-L.; Li, X.R. Research progress on siRNA delivery with nonviral carriers. Int. J. Nanomed. 2011, 6, 1017-1025. [CrossRef] [PubMed]

34. Hernandez, F.J.; Kalra, N.; Wengel, J.; Vester, B. Aptamers as a model for functional evaluation of LNA and 2'-amino LNA. Bioorg. Med. Chem. Lett. 2009, 19, 6585-6587. [CrossRef] [PubMed]

35. Karlsen, K.K.; Wengel, J. Locked nucleic acid and aptamers. Nucleic Acid Ther. 2012, 22, 366-370. [PubMed]

36. Weisbrod, S.H.; Marx, A. Novel strategies for the site-specific covalent labelling of nucleic acids. Chem. Commun. 2008, 5675-5685. [CrossRef] [PubMed]

37. Lönnberg, H. Solid-phase synthesis of oligonucleotide conjugates useful for delivery and targeting of potential nucleic acid therapeutics. Bioconjugate Chem. 2009, 20, 1065-1094. [CrossRef] [PubMed]

38. Ashwell, G.; Harford, J. Carbohydrate-specific receptors of the liver. Annu. Rev. Biochem. 1982, 51, 531-554. [CrossRef] [PubMed]

39. Spinelli, N.; Defrancq, E.; Morvan, F. Glycoclusters on oligonucleotide and PNA scaffolds: Synthesis and applications. Chem. Soc. Rev. 2013, 42, 4557-4573. [CrossRef] [PubMed]

40. Sliedregt, L.A.J.M.; Rensen, P.C.N.; Rump, E.T.; van Santbrink, P.J.; Bijsterbosch, M.K.; Valentijn, A.R.P.M.; van der Marel, G.A.; van Boom, J.H.; van Berkel, T.J.C.; Biessen, E.A.L. Design and synthesis of novel amphiphilic dendritic galactosides for selective targeting of liposomes to the hepatic asialoglycoprotein receptor. J. Med. Chem. 1999, 42, 609-618. [CrossRef] [PubMed]

41. Westerlind, U.; Westman, J.; Törnquist, E.; Smith, C.I.E.; Oscarson, S.; Lahmann, M.; Norberg, T. Ligands of the asialoglycoprotein receptor for targeted gene delivery, part 1: Synthesis of and binding studies with biotinylated cluster glycosides containing N-acetylgalactosamine. Glycoconj. J. 2004, 21, 227-241. [CrossRef] [PubMed]

42. Khorev, O.; Stokmaier, D.; Schwardt, O.; Cutting, B.; Ernst, B. Trivalent, Gal/GalNAc-containing ligands designed for the asialoglycoprotein receptor. Bioorg. Med. Chem. 2008, 16, 5216-5231. [CrossRef] [PubMed]

43. Mäkilä, J.; Jadhav, S.; Kiviniemi, A.; Käkelä, M.; Liljenbäck, H.; Poijärvi-Virta, P.; Laitala-Leinonen, T.; Lönnberg, H.; Roivainen, A.; Virta, P. Synthesis of multi-galactose-conjugated 2'-O-methyl oligoribonucleotides and their in vivo imaging with positron emission tomography. Bioorg. Med. Chem. 2014, 22, 6806-6813. [CrossRef] [PubMed]

44. Positive Initial Phase 2 Data with Revusiran (ALN-TTRsc). Available online: http:/ /www.alnylam.com/ capella/presentations/positive-initial-revusiran-phase-2-data/ (accessed on 25 April 2017).

45. Østergaard, M.E.; Yu, J.; Kinberger, G.A.; Wan, W.B.; Migawa, M.T.; Vasquez, G.; Schmidt, K.; Gaus, H.J.; Murray, H.M.; Low, A.; et al. Efficient synthesis and biological evaluation of 5 '-GalNAc conjugated antisense oligonucleotides. Bioconjugate Chem. 2015, 26, 1451-1455. [CrossRef] [PubMed] 
46. Prakash, T.P.; Wan, W.B.; Low, A.; Yu, J.; Chappell, A.E.; Gaus, H.; Kinberger, G.A.; Østergaard, M.E.; Migawa, M.T.; Swayze, E.E.; et al. Solid-phase synthesis of $5^{\prime}$-triantennary N-acetylgalactosamine conjugated antisense oligonucleotides using phosphoramidite chemistry. Bioorg. Med. Chem. Lett. 2015, 25, 4127-4130. [CrossRef] [PubMed]

47. Nair, J.K.; Willoughby, J.L.; Chan, A.; Charisse, K.; Alam, M.R.; Wang, Q.; Hoekstra, M.; Kandasamy, P.; Kel'in, A.V.; Milstein, S.; et al. Multivalent N-acetylgalactosamine-conjugated siRNA localizes in hepatocytes and elicits robust RNAi-mediated gene silencing. J. Am. Chem. Soc. 2014, 136, 16958-16961. [CrossRef] [PubMed]

48. Matsuda, S.; Keiser, K.; Nair, J.K.; Charisse, K.; Manoharan, R.M.; Kretschmer, P.; Peng, C.G.; Kel'in, A.V.; Kandasamy, P.; Willoughby, J.L.S.; et al. siRNA conjugates carrying sequentially assembled trivalent $\mathrm{N}$-acetylgalactosamine linked through nucleosides elicit robust gene silencing in vivo in hepatocytes. ACS Chem. Biol. 2015, 10, 1181-1187. [CrossRef] [PubMed]

49. Rajeev, K.G.; Nair, J.K.; Jayaraman, M.; Charisse, K.; Taneja, N.; O'Shea, J.; Willoughby, J.L.; Yucius, K.; Nguyen, T.; Shulga-Morskaya, S.; et al. Hepatocyte-specific delivery of siRNAs conjugated to novel non-nucleosidic trivalent $\mathrm{N}$-acetylgalactosamine elicits robust gene silencing in vivo. ChemBioChem 2015, 16, 903-908. [CrossRef] [PubMed]

50. Hong, V.; Presolski, S.I.; Ma, C.; Finn, M.G. Analysis and optimization of copper-catalyzed azide-alkyne cycloaddition for bioconjugation. Angew. Chem. Int. Ed. 2009, 48, 9879-9883. [CrossRef] [PubMed]

51. Ustinov, A.V.; Stepanova, I.A.; Dubnyakova, V.V.; Zatsepin, T.S.; Nozhevnikova, E.V.; Korshun, V.A. Modification of nucleic acids using [3 + 2]-dipolar cycloaddition of azides and alkynes. Rus. J. Bioorg. Chem. 2010, 36, 401-445. [CrossRef]

52. El-Sagheer, A.H.; Brown, T. Click chemistry with DNA. Chem. Soc. Rev. 2010, 39, 1388-1405. [CrossRef] [PubMed]

53. Kiviniemi, A.; Virta, P.; Lönnberg, H. Utilization of intrachain 4'-C-azidomethylthymidine for preparation of oligodeoxyribonucleotide conjugates by click chemistry in solution and on a solid support. Bioconjugate Chem. 2008, 19, 1726-1734. [CrossRef] [PubMed]

54. Gramlich, P.M.E.; Warncke, S.; Gierlich, J.; Carell, T. Click-click-click: Single to triple modification of DNA. Angew. Chem. 2008, 47, 349-3493. [CrossRef] [PubMed]

55. Berndl, S.; Herzig, N.; Kele, P.; Lachmann, D.; Li, X.; Wolfbeis, O.S.; Wagenknecht, H.-A. Comparison of a nucleosidic vs. non-nucleosidic postsynthetic "click" modification of DNA with base-labile fluorescent probes. Bioconjugate Chem. 2009, 20, 558-564. [CrossRef] [PubMed]

56. Jørgensen, A.S.; Gupta, P.; Wengel, J.; Astakhova, I.K. “Clickable” LNA/DNA probes for fluorescence sensing of nucleic acids and autoimmune antibodies. Chem. Commun. 2013, 49, 10751-10753. [CrossRef] [PubMed]

57. Astakhova, K.; Wengel, J. Interfacing click chemistry with automated oligonucleotide synthesis for the preparation of fluorescent DNA probes containing internal xanthene and cyanine dyes. Chem. Eur. J. 2013, 19, 1112-1122. [CrossRef] [PubMed]

58. Astakhova, K.; Kumar, T.S.; Campbell, M.A.; Ustinov, A.V.; Korshun, V.A.; Wengel, J. Branched DNA nanostructures efficiently stabilised and monitored by novel pyrene-perylene 2 '- $\alpha$ - $L$-amino-LNA FRET pairs. Chem. Commun. 2013, 49, 511-513. [CrossRef] [PubMed]

59. Astakhova, K.; Hansen, L.H.; Vester, B.; Wengel, J. Peptide-LNA oligonucleotide conjugates. Org. Biomol. Chem. 2013, 11, 4240-4249. [CrossRef] [PubMed]

60. Aertner, L.M.; Merkel, L.; Bohlke, N.; Braun, F.B.; Weise, C.; Dernedde, J.; Budisa, N.; Hackenberger, C.P.R. Site-selective modification of proteins for the synthesis of structurally defined multivalent scaffolds. Chem. Commun. 2012, 48, 522-524. [CrossRef] [PubMed]

61. Chang, T.; Lai, C.; Chien, C.; Liang, C.; Adak, A.K.; Chuang, Y.; Chen, Y.; Lin, C. Synthesis and evaluation of a photoactive probe with a multivalent carbohydrate for capturing carbohydrate-lectin interactions. Bioconjugate Chem. 2013, 24, 1895-1906. [CrossRef] [PubMed]

62. Kikkeri, R.; Liu, X.; Adibekian, A.; Tsaia, Yu-H.; Seeberger, P.H. Facile synthesis of size dependent $\mathrm{Ru}(\mathrm{II})$-carbohydrate dendrimers via click chemistry. Chem. Commun. 2010, 46, 2197-2199. [CrossRef] [PubMed]

63. Lou, C.; Vester, B.; Wengel, J. Oligonucleotides containing a piperazino-modified 2'-amino-LNA monomer exhibit very high duplex stability and remarkable nuclease resistance. Chem. Commun. 2015, 51, 4024-4027. [CrossRef] [PubMed] 
64. Nabo, L.J.; Madsen, C.S.; Jensen, K.J.; Kongsted, J.; Astakhova, K. Ultramild protein-mediated click chemistry creates efficient oligonucleotide probes for targeting and detecting nucleic acids. ChemBioChem 2015, 16, 1163-1167. [CrossRef] [PubMed]

65. Ries, A.; Kumar, R.; Lou, C.; Kosbar, T.; Vengut-Climent, E.; Jørgensen, P.T.; Morales, J.C.; Wengel, J. Synthesis and biophysical investigations of oligonucleotides containing galactose-modified DNA, LNA, and 2'-amino-LNA monomers. J. Org. Chem. 2016, 81, 10845-10856. [CrossRef] [PubMed]

66. Lou, C.; Samuelsen, S.V.; Christensen, N.J.; Vester, B.; Wengel, J. Oligonucleotides containing aminated 2 -amino-LNA nucleotides: Synthesis and strong binding to complementary DNA and RNA. Bioconjugate Chem. 2017, 28, 1214-1220. [CrossRef] [PubMed]

67. Patel, A.; Lindhorst, T.K. A modular approach for the synthesis of oligosaccharide mimetics. J. Org. Chem. 2001, 66, 2674-2680. [CrossRef] [PubMed]

68. Kværnø, L.; Kumar, R.; Dahl, B.M.; Olsen, C.E.; Wengel, J. Synthesis of abasic locked nucleic acid and two seco-LNA derivatives and evaluation of their hybridization properties compared with their more flexible DNA counterparts. J. Org. Chem. 2000, 65, 5167-5176. [CrossRef] [PubMed]

Sample Availability: Not available. 